\title{
Sexual Polarity in Streptomyces coelicolor
}

\author{
By G. SERMONTI AND SANDRA CASCIANO \\ Research Unit for Microbial Genetics, Istituto Superiore di Sanità, \\ Rome, Italy
}

(Received 8 May 1963)

SUMMARY

A group of intersterile strains $\left(\mathbf{R}^{-}\right)$have been identified in Streptomyces coelicolor Strain A $3(2)$. They give fertile crosses with other strains $\left(\mathrm{R}^{+}\right)$ which in turn are fertile among themselves. In $\mathbf{R}^{-} \times \mathbf{R}^{+}$crosses the contribution of $\mathbf{R}^{+}$markers to the recombinant progeny is usually small. The fertility factor(s), which cannot be eliminated by acridine dyes, may be transferred at high frequency in $\mathrm{R}^{+} \times \mathrm{R}^{-}$crosses, but the fertilized strains display a low fertility.

\section{INTRODUCTION}

Practically all the bacteria whose conjugation has been investigated have shown sexual polarity, that is, differentiation into donor (male) and recipient (female) strains. This phenomenon has usually been first observed when, after the performance of numerous crosses, some attempted cross has failed to give recombinant progeny (Lederberg, Cavalli \& Lederberg, 1952). The sterility of a cross may be caused by various genetical as well as environmental factors, but as a rule it has been shown to be due to an encounter between two recipient strains.

Sexual polarity is manifested in various ways: the donor strain contributes fewer markers to the progeny (Hayes, 1952; Cavalli-Sforza, Lederberg \& Lederberg, 1953); the donor strain may infect the recipient with its fertility factor (Lederberg $e t a l$. 1952; Hayes, 1953).

In Streptomyces coelicolor Bradley \& Anderson (1958) reported the occurrence of some sterile crosses, but they gave no indication of the causes of the observed sterility. Hopwood \& Sermonti (1962) and Sermonti \& Hopwood (1964) observed in $S$. coelicolor A $3(2)$ the occurrence of several sterile combinations of strains and a group of strains fertile in all combinations. This study extends these preliminary observations and in particular is concerned with the analysis of reciprocal crosses between strains of opposite 'sex'.

\section{METHODS}

The routine techniques used in the genetics of Streptomyces coelicolor were described in detail in a review paper by Hopwood \& Sermonti (1962).

Strains. All the strains used in this work derive from the wild type of Streptomyces coelicolor A3 (2) and have been obtained either as mutants after ultraviolet (u.v.) or X-ray treatment, or as recombinants from crosses between mutant strains. The symbols and phenotypes of the mutant alleles present in the strains used are shown 
in Table 1. The order of the markers used here and the distances between them on the two linkage groups are as follows (Hopwood \& Sermonti, 1962; Hopwood, personal communication, for location of leu-1):

$$
\text { I } \frac{l \quad m \quad h \quad a}{8 \quad 3 \quad 11} ; \quad \text { II } \frac{u p p s}{9} \text {. }
$$

The order of the markers in linkage group II with respect to those in group $I$ is reversed as compared with the order arbitrarily chosen by Hopwood (1959) and adopted in later papers, for simplicity of description of some data.

Table 1. Symbols and origins of the strains of Streptomyces coelicolor used

Strains 4 and 8 are mutants of the wild type; these, and strains 109,118 and 82 , which are recombinants, were kindly supplied by Dr D. A. Hopwood. Strains 53, 44 and 43 are recombinants from the same cross $(47 h p s \times 15 \mathrm{~m})$. Strain 87 is a segregant from cross $109 a u \times 47$ hps.

The meanings of the symbols are as follows: arg $(a)$, requirement of arginine; his $(h)$, requirement of histidine; met $(m)$, requirement of methionine; leu $(l)$, requirement of leucine; phe $(p)$, requirement of phenylalanine; ura $(u)$, requirement of uracil; str $(s)$, resistance to streptomycin.

\begin{tabular}{|c|c|c|c|c|c|}
\hline \multicolumn{6}{|c|}{ Fertility type } \\
\hline \multicolumn{3}{|c|}{$\mathbf{R}^{+}$} & \multicolumn{2}{|r|}{$\mathbf{R}^{-}$} & \\
\hline $\begin{array}{c}\text { Code } \\
\text { no. }\end{array}$ & Genotype & $\begin{array}{l}\text { Abbreviated } \\
\text { symbols }\end{array}$ & $\begin{array}{l}\text { Code } \\
\text { no. }\end{array}$ & Genotype & $\begin{array}{c}\text { Abbreviated } \\
\text { symbols }\end{array}$ \\
\hline 4 & his-1 & $h$ & 44 & his-1 & $h$ \\
\hline 53 & met-2, phe-1, str-1 & $m p s$ & 43 & met-2, his-1, str-1 & $m h s$ \\
\hline 87 & arg- 1, ura- 1, str -1 & aus & 118 & arg- 1, ura-1, str-1 & aus \\
\hline 109 & $\arg -1$, ura-1 & $a u$ & 8 & his-12 & h12 \\
\hline 82 & leu-1, met-2, arg- $1, s$ & lmasp & & & \\
\hline
\end{tabular}

Media. A minimal medium (glucose asparagine agar) and two complete media were used; the latter are a sporulation medium and a reproduction medium (medium 1, according to Hopwood \& Sermonti, 1962).

Crosses. Crosses were carried out by mixing the spores of two strains on slopes of reproduction medium, harvesting the spores of the mixed cultures after three or four days and plating these on selective media (minimal media variously supplemented). Characterization of the recombinants was done by replica-plating on diagnostic media.

Fertility tests. Various procedures have been adopted. The one most commonly used was as follows. Spores of the tester strain (usually 'sterile', see later) were streaked with a loop on a large area of a Petri dish containing well-dried reproduction agar medium. Spores of two or three strains to be tested were streaked separately side by side across the streak of the tester strain (Pl. 1). After incubation for 3-4 days the mixed culture was replica-plated on selective media: two media each selective for one of the parents, and one or more media selective for recombinants. The cross was considered fertile when on the latter media at least 24, recombinant colonies appeared in the area corresponding to the mixed culture, and sterile when recombinants were entirely absent and this sterility could not be attributed to imbalance between the two parent strains or to very 
close linkage between the selected markers. Some 'sterile' crosses gave rise sometimes to a few minute colonies which turned out to be syntrophic growths (Pl. 1).

A more quantitative sterility diagnosis was obtained when the spores of an attempted cross performed by the standard procedure, then plated at high densities (at least $10^{6} \mathrm{spores} / \mathrm{dish}$ ) on a medium selective for unlinked markers, gave rise to no or very few recombinants. 'Fertile' crosses gave recombinants with frequencies of the order of $10^{-4}$ to $10^{-3}$ plated spores. These values appeared in approximately balanced mixed cultures (Table 2). The fertility test was always affected by some degree of uncertainty and subjectivity. In the present work strains have been used which showed the most extreme characteristics as regards the fertility test (Table 1).

Table 2. Fertility and sterility in arg-1, ura-1 (str-1*) $\times$ his-1 crosses of Streptomyces coelicolor mutants

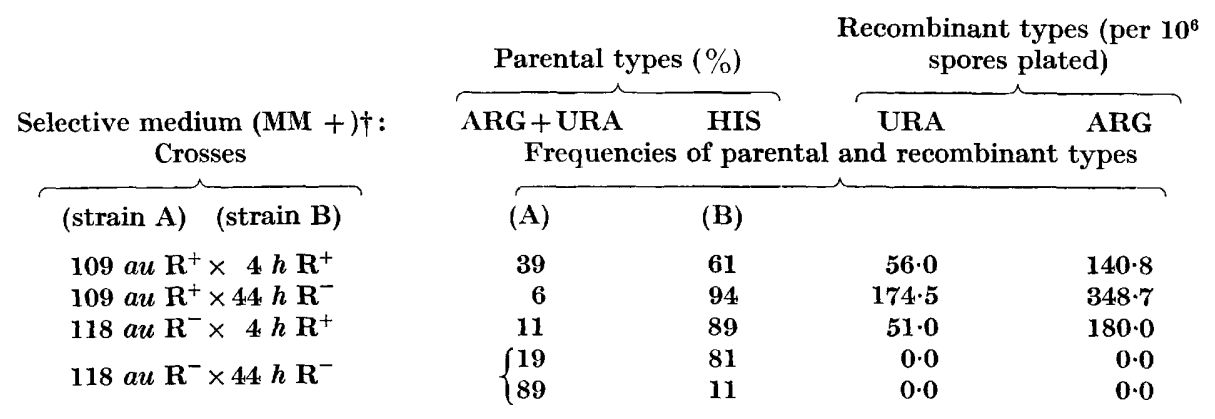

* The character $s i r-1$ has been ignored. Strain 109 is sensitive to streptomycin, strain 118 is resistant to streptomycin. Strain 87 aus $\mathbf{R}^{+}$gives results similar to 109 au.

$\dagger$ The substances added to the minimal media (MM) are indicated with the same symbols used to indicate the requirements (see Table 1; or resistance, Table 4), but in capital letters.

\section{RESULTS}

\section{Fertile and sterile crosses}

Some strains of Streptomyces coelicolor A 3 (2), when crossed with one another, did not produce any recombinant progeny or gave rise only to a very small number of recombinants (less than $1 / 10^{6}$ plated spores) on given selective media. The sterility diagnosis (see Methods) was, however, sometimes not absolute since crosses which did not give rise to any recombinants on one selective medium sometimes gave a small number of recombinants on another medium. Moreover, a combination of strains which had been sterile in some crosses could, after a few months storage of the strains, become moderately fertile. However, it has been possible to collect a small group of strains which gave the most regular negative response, which has been called $\mathbf{R}^{-}(\mathbf{R}=$ recombination $)$. These gave no or very few recombinants when crossed with one another, but were fertile when crossed with any other strain $\left(\mathbf{R}^{+}\right)$. Crosses between the various $\mathbf{R}^{+}$strains were also regularly fertile. The situation may therefore be summarized as follows: $\mathbf{R}^{-} \times \mathbf{R}^{-}=$sterile or semi-sterile; $\mathbf{R}^{-} \times \mathbf{R}^{+}=$fertile; $\mathbf{R}^{+} \times \mathbf{R}^{+}=$fertile. A range of strains with a degree of fertility intermediate between that of the $\mathbf{R}^{-}$and $\mathbf{R}^{+}$strains used in this work (Table 1 ) probably exists. 


\section{Analysis of crosses between $\mathrm{R}^{+}$and $\mathrm{R}^{-}$strains}

In many bacteria, and in particular in Escherichia coli $\mathrm{k} 12$, the alleles of the male parent appear in the progeny with frequencies considerably lower than those of the alleles of the female parent (Hayes, 1953). In $H f r \times \mathrm{F}^{-}$crosses the larger the distance between an allele of the $\mathrm{Hfr}$ strain and the origin of the chromosome the smaller is the frequency of this allele among recombinants (Wollman, Jacob \& Hayes, 1956). However, estimation of allele ratios is made difficult by the obligatory use of selected markers which will appear in the progeny with frequencies of $100 \%$, affecting the frequency of linked alleles. Even when an unselected marker is not linked with a selected one, the allele ratio at the first locus varies according to whether the selected $H f r$ marker precedes or follows the selected marker in the process of transfer.

In Streptomyces coelicolor there are two linkage groups which appear to segregate independently. Hopwood \& Sermonti (1962) gave good evidence indicating that these might correspond to two distinct chromosomes. We shall therefore provisionally assume that these are independent also in the process of transfer and integration. On this assumption, by selecting markers on one linkage group, we can obtain an estimate of the allele ratio at the loci on the other linkage group, among the recombinants from the mixed culture. In the present paper we shall mainly consider four-point crosses with two selectable alleles in repulsion in each linkage group. Recombinants have been detected on two media, on each of which two alleles in one linkage group were selected and the alleles on the other were non-selected and therefore available for analysis, which has been carried out by the routine procedure.

For each pair of linked genes four segregant classes occur, which are indicated in Table 3 by the four combinations of two symbols, $\mathbf{R}^{+}$and $\mathbf{R}^{-} \cdot \mathbf{R}^{-} \mathbf{R}^{-}$indicates the parental class with the $\mathbf{R}^{-}$alleles; $\mathbf{R}^{+} \mathbf{R}^{+}$the one with the $\mathbf{R}^{+}$alleles; $\mathbf{R}^{+} \mathbf{R}^{-}$the recombinant class deriving the left-hand (see map in Methods) allele from the $\mathbf{R}^{+}$ parent and the right-hand allele from the $\mathbf{R}^{-}$parent; $\mathbf{R}^{-} \mathbf{R}^{+}$the complementary class. If we consider, for instance, the first linkage group in cross $\mathrm{R}^{+} h \times \mathrm{R}^{-}$aus :

$$
\frac{\mathbf{R}^{+}}{\mathbf{R}^{-}} \frac{h+}{+a}
$$

The four combinations of symbols will correspond to the four combinations of markers as follows:

$$
\begin{array}{lll}
\mathbf{R}^{-} \mathbf{R}^{-} & + & a \\
\mathbf{R}^{+} \mathbf{R}^{+} & h & + \\
\mathbf{R}^{+} \mathbf{R}^{-} & h & a \\
\mathbf{R}^{-} \mathbf{R}^{+} & + & +
\end{array}
$$

The data in Table 3 permit the same kind of treatment that was developed in the analysis of heteroclones (Hopwood \& Sermonti, 1962; Hopwood, Sermonti \& SpadaSermonti, 1963; Sermonti \& Hopwood, 1964) except for the test of independence between the two linkage groups, which cannot be made. The almost regular excess of the $\mathbf{R}^{-} \mathbf{R}^{-}$class in respect to the $\mathbf{R}^{+} \mathbf{R}^{+}$class points to a disturbance affecting the chromosomes derived from the $\mathbf{R}^{+}$parent, while the excess of the $\mathbf{R}^{+} \mathbf{R}^{-}$class in 
respect to the $\mathbf{R}^{-} \mathbf{R}^{+}$indicates a disturbance on the right side of the $\mathbf{R}^{+}$chromosome (probably accompanied in some cases by a disturbance on the left side of the $\mathbf{R}^{-}$ chromosome).

\section{'Reciprocal' crosses $\mathbf{R}^{+} \times \mathbf{R}^{-}$}

The occurrence of strains identical as regards the genetic markers and behaving differently in the fertility test (Table 1 ) allows the performance of reciprocal crosses, for instance two crosses between strains aus and $h$, in one of which the aus strain is the $\mathbf{R}^{-}$and in the other the $\mathbf{R}^{+}$. Since the cause of sterility and fertility in various strains is not yet known, and sterility (or fertility) of the various strains may be due to different conditions, the reciprocity of crosses may be stated only with a certain degree of approximation. However, the data in Table $\mathbf{3}$ clearly show that $\mathbf{R}^{+}$ markers are generally in defect. Out of a total of six crosses, implying analysis of twelve chromosomes, ten chromosomes have the male markers in a more or less pronounced minority (Table 3). The data in Table 3, first two columns, re-elaborated in Table 4 to make the position of the unselected markers more evident, clearly show that the different frequencies of the alleles cannot be attributed to the linkage of the unselected markers with the selected ones. In fact, the selective conditions are identical in the reciprocal crosses, while the ratio of the frequency among the $\mathbf{R}^{+}$ and $\mathbf{R}^{-}$alleles of the unselected linkage group is inverted. The deficiency of the $\mathbf{R}^{+}$markers must therefore be attributed to some other factor, most probably the fertility factor.

Table 3. Frequencies of allele combinations at pairs of linked loci*, reciprocal crosses

Crosses $\dagger \quad \ldots$

\begin{tabular}{|c|c|c|}
\hline $\begin{array}{l}\text { 'Sex' of } \\
\text { parents' }\end{array}$ & \multicolumn{2}{|c|}{$\begin{array}{l}\text { Allele combinations } \\
\text { (origin of alleles } \$ \text { ) }\end{array}$} \\
\hline $\mathbf{R}^{+}$ & Parental & $\left\{\begin{array}{l}\mathbf{R}^{-} \mathbf{R}^{-} \\
\mathbf{R}^{+} \mathbf{R}^{+}\end{array}\right.$ \\
\hline$\overline{\mathbf{R}^{-}}$ & Recombinant & $\left\{\begin{array}{l}\mathbf{R}^{+} \mathbf{R}^{-} \\
\mathbf{R}^{-} \mathbf{R}^{+}\end{array}\right.$ \\
\hline $\mathbf{R}^{-}$ & Parental & $\left\{\begin{array}{l}\mathbf{R}^{-} \mathbf{R}^{-} \\
\mathbf{R}^{+} \mathbf{R}^{+}\end{array}\right.$ \\
\hline$\overline{\mathbf{R}^{+}}$ & Recombir & $\left\{\begin{array}{l}\mathbf{R}^{+} \mathbf{R}^{-} \\
\mathbf{R}^{-} \mathbf{R}^{+}\end{array}\right.$ \\
\hline
\end{tabular}

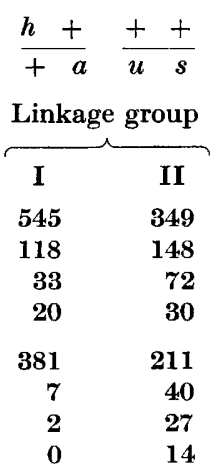

$\frac{m+}{+a} \frac{+p(s)}{u+(s)}$

Linkage group

$\begin{array}{rr}\text { I } & \text { II } \\ 136 & 153 \\ 5 & 1 \\ 0 & 1 \\ 0 & 0 \\ 76 & 71 \\ 45 & 101 \\ 0 & 16 \\ 0 & 4\end{array}$

Linkage group

\begin{tabular}{rr}
\hline I & II \\
175 & 171 \\
19 & 10 \\
7 & 10 \\
6 & 4 \\
102 & 39 \\
43 & 178 \\
2 & 16 \\
1 & 0
\end{tabular}

* The segregation of markers of linkage group I was studied on a medium selective for markers of linkage group II and vice versa (see Table 4).

$t$ The alleles of the parent strains are written as in the presumptive zygotes, one parent above and the other below the lines, which refer to the two chromosomes.

‡ The symbol above the line refers to the parent strain written above the lines beside crosses.

$\S$ The symbols $\mathbf{R}^{+}$and $\mathbf{R}^{-}$indicate the parent from which each allele derives. The first symbol refers to the left-hand allele of either linkage group, the second to the right-hand one (see text).

In two crosses, both involving the strain $m p s \mathbf{R}^{-}$, the $\mathbf{R}^{-}$alleles on chromosome II are in a minority (Table 3; see figures in italics). The $\mathbf{R}^{+} \mathbf{R}^{-}$recombinants are still in excess over the complementary ones. In both cases the defect may be attributed to a single disturbance to the left end of the $\mathbf{R}^{-}$chromosome, perhaps accompanied by a less pronounced disturbance to the right end of the $\mathbf{R}^{+}$chromosome. 
Table 4. Allele ratios in reciprocal crosses*

\begin{tabular}{|c|c|c|c|c|c|c|c|c|c|c|c|}
\hline \multicolumn{4}{|c|}{ Selective media $(M M+) \dagger$} & \multicolumn{4}{|c|}{ HIS + ARG + STR } & \multicolumn{4}{|c|}{ URA } \\
\hline & & & & \multicolumn{8}{|c|}{ Frequency of alleles $(\%) \div$} \\
\hline \multirow{6}{*}{ Chromosome } & \multirow{3}{*}{$\ldots$} & \multicolumn{2}{|c|}{ Crosses $§$} & \multirow{2}{*}{\multicolumn{2}{|c|}{$\begin{array}{l}\text { Unselected } \\
\text { markers } \\
\text { I }\end{array}$}} & \multirow{2}{*}{\multicolumn{2}{|c|}{$\begin{array}{c}\text { Selected } \\
\text { markers } \\
\quad \text { II }\end{array}$}} & \multirow{2}{*}{\multicolumn{2}{|c|}{$\begin{array}{c}\text { Selected } \\
\text { markers } \\
\text { I }\end{array}$}} & \multirow{2}{*}{\multicolumn{2}{|c|}{$\begin{array}{c}\text { Unselected } \\
\text { markers } \\
\text { II }\end{array}$}} \\
\hline & & $\mathbf{I}$ & II & & & & & & & & \\
\hline & & $h+$ & ++ & 25 & 6 & 100 & $\mathbf{0}$ & 0 & 100 & 37 & 30 \\
\hline & $\overline{\mathbf{R}^{-}}$ & $\overline{+a}$ & $\overline{u s}$ & 75 & $\overline{94}$ & 0 & 100 & 100 & $\overline{0}$ & 63 & 70 \\
\hline & $\mathbf{R}^{-}$ & $h+$ & ++ & 97 & 98 & 100 & $\mathbf{0}$ & $\mathbf{0}$ & 100 & 77 & 82 \\
\hline & $\overline{\mathbf{R}^{+}}$ & $\overline{+a}$ & $\overline{u s}$ & 3 & $\overline{2}$ & $\mathbf{0}$ & 100 & 100 & $\overline{0}$ & $\overline{\mathbf{2 3}}$ & 18 \\
\hline
\end{tabular}

* Detailed analysis in Table 3.

$\dagger$ See note to Table 2.

$\ddagger$ Each number represents the frequency of the allele in the corresponding position (under 'crosses').

$\S$ The alleles of the parent strains are written as in the presumptive zygotes, one parent above and the other below the lines, which refer to the two chromosomes.

In several cases when two strains differ in more than two markers on one linkage group, it is observed that the $\mathrm{R}^{+}$markers show frequencies gradually decreasing from the left to the right, in agreement with what is found in the four-point crosses. Table 5 shows an example in linkage group I. The order of the frequencies of the $\mathbf{R}^{+}$alleles corresponds very well to the order of the genes along the linkage group.

Table 5. Gradient in allele frequencies on linkage group I

Cross $\quad \ldots \quad 82$ leu-1 met-2 arg-1 str-1 phe-1 $\mathbf{R}^{+} \times 8$ his-12 $\mathbf{R}^{-}$

Selective medium: $(\mathrm{MM}+)^{*} \quad \ldots \quad \mathrm{LEU}+\mathrm{MET}+\mathrm{HIS}+\mathrm{ARG}+\mathrm{STR}$

\begin{tabular}{cccc} 
& & & \\
& & & \\
\multicolumn{4}{c}{ Genotype } \\
+ & + & $h$ & + \\
$l$ & $m$ & + & $a$ \\
$l$ & + & $h$ & + \\
+ & $m$ & + & $a$ \\
$l$ & $m$ & $h$ & + \\
+ & + & + & $a$ \\
$l$ & $m$ & + & + \\
+ & + & $h$ & $a$
\end{tabular}

Cross-

\section{over in}

no. region

Genotype

Cross-over

$\begin{array}{rr}448 & - \\ 6 & - \\ 36 & 1 \\ 1 & 1 \\ 2 & 2 \\ 0 & 2 \\ 15 & 3 \\ 0 & 3\end{array}$

Regions

$\begin{array}{rrrr}+ & m & + & + \\ l & + & h & a\end{array}$

$+m h+$

$l++a$

$++++$

$l \quad m \quad h \quad a$

$l+++$

$+m h a$

no.

in region

Allele ratios

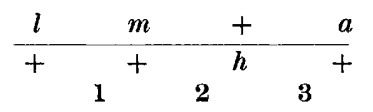

1,3

1,3

1,2

1,2

2, 3

2, 3

$1,2,3$

$1,2,3$

\begin{tabular}{rrrr}
59 & 36 & 32 & 8 \\
\hline 461 & 484 & 488 & 512
\end{tabular}

* See note to Table 2. 


\section{Transmissibility of the $\boldsymbol{R}$ factor}

Only a few preliminary data bearing on this point have been collected. Sterility segregates in some crosses, and in fact several of the $\mathbf{R}^{-}$strains used in this work were obtained as recombinants from crosses, together with $\mathbf{R}^{+}$strains (Table 1 ). The large majority of segregants is, however, of the $\mathbf{R}^{+}$type (Hopwood \& Sermonti, 1962). The fertility factor may be transferred at high frequency in $R^{+} \times \mathbf{R}^{-}$crosses (Hopwood \& Sermonti, 1962). Strains made fertile in this way, however, show a low degree of fertility and in crosses with $\mathbf{R}^{-}$strains the markers of the fertilized parent do not show any clear tendency to be less frequent. Nothing is yet known about the nature of the fertility factor. We have frequently observed that sterile strains become moderately fertile with time, requiring continuous purification to preserve the character. All attempts to eliminate the $\mathbf{R}$ factor by means of acridine dyes, which have been effective in Escherichia coli (Hirota, 1960), have been unsuccessful.

\section{DISCUSSION}

The compatibility system in Streptomyces coelicolor, like that of the other bacteria showing sexual differentiation, involves a group of intersterile strains $\left(\mathbf{R}^{-}\right)$and a group of strains fertile in all combinations $\left(\mathbf{R}^{+}\right)$. By analogy with Escherichia coli $\mathrm{K} 12$ (Hayes, 1953) we may suppose that the strains of the first group act as recipients (females) and those of the second as donors (males). The latter may presumably act also as recipients in $\mathbf{R}^{+} \times \mathbf{R}^{+}$crosses. The only evidence of a sexual polarity is based on the different contributions to the progeny of the markers of the two 'sexes'. The $\mathbf{R}^{+}$markers, as a rule, are less frequent. In this respect also $S$. coelicolor behaves in the same way as other bacteria (for review see Jacob \& Wollman, 1961). The deficiency of the $\mathrm{R}^{+}$markers may be attributed to the incompleteness of the male chromosomes at their right ends, in all the $\mathbf{R}^{+}$strains examined so far.

The occurrence of a gradient in the frequencies of $\mathrm{R}^{+}$alleles, decreasing from left to right, points to a situation corresponding to that in $H f r$ strains in Escherichia coli $\mathbf{k} 12$. Occasionally the $\mathbf{R}^{-}$markers are in defect. In this case the deficiency may be attributed to a loss at the left end of chromosome II, i.e. at the end opposite that at which the deficiencies in the male chromosomes are observed. This may be due to a tendency of the defective female chromosome to become incomplete at the left end, perhaps as a consequence of a post-zygotic loss. This shortage of markers of the recipient strains was observed by Lederberg (1949) in heterozygous strains of E. coli $\mathrm{K} 12$ and by Smith \& Stocker (1962) in Salmonella typhimurium.

The infective nature of the fertility factor is far from clear. In $\mathbf{R}^{+} \times \mathbf{R}^{-}$mixed cultures it seems transmissible at high frequency, but the newly fertile strains display only a low fertility. This situation has a parallel in Pseudomonas aeruginosa (Holloway \& Fargie, 1960). The failure of acridine dyes to render the $\mathbf{R}^{+}$strains infertile is further evidence of the integrated nature of the fertility factor in $\mathbf{R}^{+}$ strains. Moreover, although the behaviour of the $\mathbf{R}$ factor in the progeny of crosses has not yet been extensively studied, there is some evidence that $\mathbf{R}^{-}$strains and $\mathbf{R}^{+}$strains may be produced among recombinants of the same cross, thus indicating the possible segregation of the character. However, the fertility factor can pre- 
sumably sometimes be lost spontaneously, or following irradiation, since all strains of Streptomyces coelicolor A $3(2)$ used in genetical studies, both $\mathbf{R}^{+}$and $\mathbf{R}^{-}$, were derived from a single original culture (Hopwood, 1959), which had been purified at least once by the isolation of a single spore.

In conclusion we cannot yet decide whether the fertility factor(s) occur(s) in the free or in the integrated state in Streptomyces coelicolor, both conditions being probably present in the different strains. Since Streptomyces coelicolor has two linkage groups and perhaps two chromosomes, it will be of great interest to establish whether one or two fertility factors are involved in the process of genetic transfer and whether the markers of the two linkage groups behave independently during this process.

This work was aided by a grant from the Italian Consiglio Nazionale delle Ricerche (C.N.R.). The authors wish to express their thanks to Dr B. Fratello and Mrs Isabella Spada-Sermonti for allowing the use of some of their unpublished data and to Dr D. A. Hopwood (University of Glasgow) for the revision of the manuscript and for useful suggestions.

\section{REFERENCES}

Bradley, S. G. \& Anderson, D. L. (1958). Compatibility system controlling heterokaryon formation in Streptomyces coelicolor. Proc. Soc. exp. Biol., N.Y. 99, 476.

Cavalui-Sforza, L. L., Lederberg, J. \& Lederberg, E. (1953). An infective factor controlling sex compatibility in Bacterium coli. J. gen. Microbiol. 8, 89.

HaYes, W. (1952). Recombination in Bacterium coli $\mathrm{k} 12$ : unidirectional transfer of genetic material. Nature, Lond. 169, 118.

HAYES, W. (1953). Observations on a transmissible agent determining sexual differentiation in Bacterium coli. J. gen. Microbiol. 8, 72.

Hirota, Y. (1960). The effect of acridine dyes on mating type factors in Escherichia coli. Proc. nat. Acad. Sci., Wash. 46, 57.

Holloway, B. W. \& Fargie, B. (1960). Fertility factors and genetic linkage in Pseudomonas aeruginosa. J. Bact. 80, 362.

Hopwood, D. A. (1959). Linkage and the mechanism of recombination in Streptomyces coelicolor. Ann. N.Y. Acad. Sci. 81, 887.

Hopwood, D. A. \& Sermonti, G. (1962). The genetics of Streptomyces coelicolor. Advanc. Genet. 11, 273.

Hopwood, D. A., Sermonti, G. \& Spada-Sermonti, I. (1963). Heterozygous clones in Streptomyces coelicolor. J. gen. Microbiol. 30, 249.

Jacob, F. \& Wollman, E. L. (1961). Sexuality and the Genetics of Bacteria. New York: Academic Press.

Lederberg, J. (1949). Aberrant heterozygotes in Escherichia coli. Proc. nat. Acad. Sci., Wash. 35, 178.

Lederberg, J., Cavalui, L. L. \& Lederberg, E. M. (1952). Sex compatibility in Escherichia coli. Genetics, 37, 720.

Sermonti, G. \& Hopwood, D. A. (1964). Recombination in Streptomyces coelicolor, in The Bacteria, Biochemistry, Genetics and Physiology, Vol. 5. New York: Academic Press (in the Press).

Sмrth, S. \& Stocker, B. A. D. (1962). Colicinogeny and recombination. Brit. med. Bull. $18,46$.

Wollman, E. L., JAcob, F. \& HAYes, W. (1956). Conjugation and genetic recombination in Escherichia coli. Cold Spr. Harb. Symp. quant. Biol. 21, 141. 


\section{EXPLANATION OF PLATE}

\section{Fertility test in Streptomyces coelicolor}

Fig. 1. Mixed cultures on reproduction medium: the tester strain (118 arg-1 ura-1 str-1 $\mathrm{R}^{-}$) was streaked first and the three strains to be tested (all requiring histidine; from the left, two $R^{+}$and one $\mathbf{R}^{-}$) were cross-streaked on the tester strain.

Fig. 2. Replica plate of the mixed cultures on to a medium supporting growth of the tested strain : minimal medium (MM) + histidine.

Fig. 3. Replica plate of the mixed cultures on to a medium supporting growth of the tester strain : MM + arginine + uracil.

Fig. 4. Replica plate of the mixed cultures on to a medium supporting growth of recombinants only: MM+arginine.

The white lines have been drawn to mark the zones corresponding to the tester and the tested strains. Observe recombinant colonies in the two fertile crosses (left and centre), while only sparse traces of abortive growth derive from the 'sterile' cross (right). 

Journal of General Microbiology, Vol. 33, No. 2

Plate 1

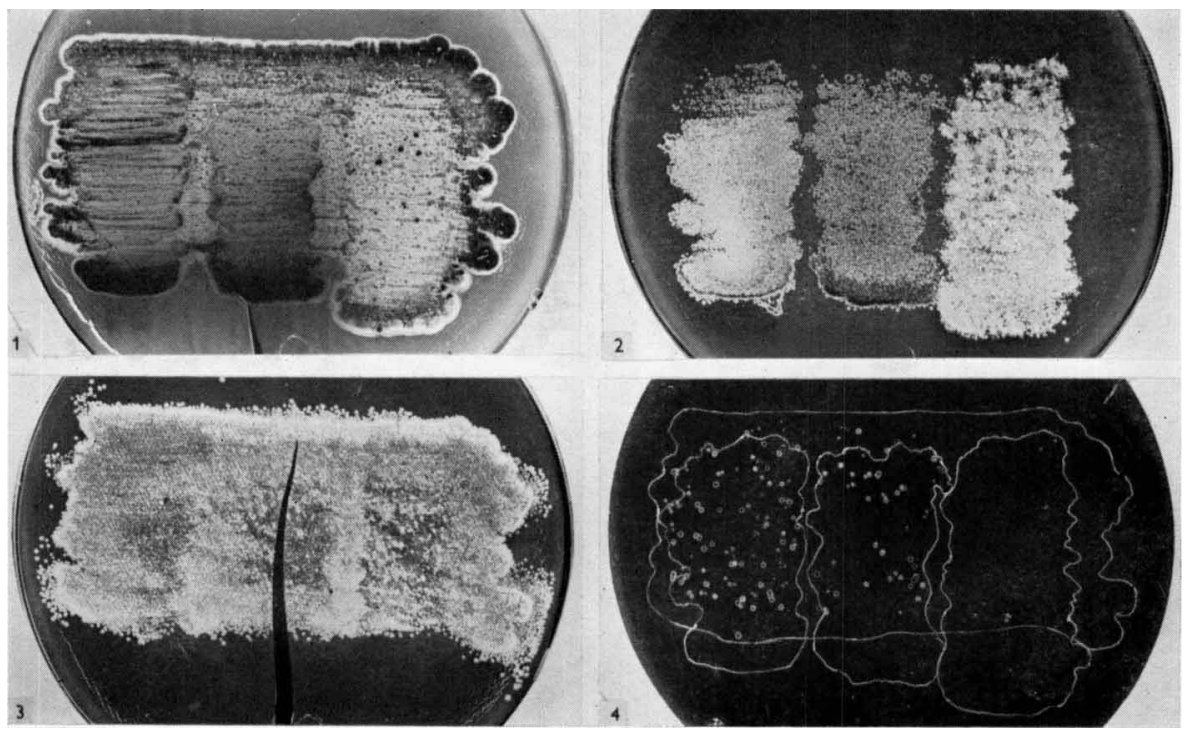

